CIENCIA Y SOCIEDAD

Volumen XXX, Número 2

Enero-Marzo 2005

\title{
¿ES LA DOLARIZACIÓN OFICIAL UNA OPCIÓN REAL PARA LAS ECONOMÍAS EMERGENTES?
}

Alexis Cruz*

\section{RESUMEN}

Este trabajo analiza los costos y beneficios para una economía emergente de adoptar oficialmente la dolarización. La magnitud de los costos y beneficios involucrados en dicho proceso no serán iguales para todos los países y dependerán de la característica particular de cada economía, así como también de su relación con el país propietario de la moneda y con otros países oficialmente dolarizados con la misma moneda. Los países que adoptan la dolarizacion total de su economía obtienen importantes beneficios como bajas tasa de inflación y bajas tasas de interés pero no está claro que tenga un impacto positivo en el crecimiento económico. El grado de dolarización parcial que tenga la economía antes de iniciar una dolarización total juega un papel clave para determinar los costos de la medida.

PALABRAS CLAVES

Dolarización, tipo de cambio, unión monetaria.

\section{Introducción}

En el último tiempo, el régimen de tipo de cambio ha sido identificado como un factor de crisis financiera en la mayoría

* Agradezco los comentarios de Alexandro Mandilaras, Virgilio Rodríguez, Graham Bird, Paul Levine y los asistentes a los seminarios del Macroeconomy Study Group de la Universidad de Surrey. La dirección del autor es: Departament of Economics, University of Surrey, Guildford, GU2 7XH, UK. Email: a.cruz@surrey.ac.uk 
de los mercados emergentes -México a fines de 1994; Tailandia, Indonesia y Korea en 1997; Rusia y Brazil en 1998; Argentina y Turquía en 2000; y Turquía otra vez en 2001. Junto con desatarse las crisis monetarias de Asia y Rusia en $1997-$ 98 se renovó el debate teórico sobre cuál será el régimen cambiario óptimo que ayude a las economías emergentes a evitar contagios financieros y crisis monetarias, y que a su vez les permita alcanzar credibilidad y crecimiento. Muchos economistas sugieren que estos países deben adoptar una moneda supranacional fuerte como una forma de alcanzar los objetivos anteriormente señalados. La adopción de esa fuerte moneda supranacional ha sido llamada dolarización oficial.

El término dolarización oficial entró al debate económico en el año 1999 cuando Argentina fue afectada por la incertidumbre provocada por la suspensión de pagos (default) de Rusia y la devaluación brasileña. Ante esa situación, el expresidente Menen anunció oficialmente que su país estaba considerando reemplazar el peso por el dólar de los Estados Unidos. Hasta esa fecha sólo 14 países independientes estaban oficialmente dolarizados', siendo Panamá el país con el período de dolarización más largo, al haber adoptado el dólar de los Estados Unidos para reemplazar su moneda nacional en el año 1904.

En el año 2000, Ecuador pasó a formar parte de los países oficialmente dolarizados al adoptar el dólar de los Estados Unidos como su moneda oficial, siendo seguido por El Salvador en el 2001. Hasta el momento, Guatemala y Nicaragua

1 Andorra, Chipre Septentrional (independiente de facto), Timor Oriental, Kiribati, Liechtenstein. Islas Marshall, Micronesia, Mónaco, Nauru, Palau, Panamá, San Marino, Tuvalu y Santa Sede (Vaticano). 
han adoptado informalmente el dólar de los Estados Unidos como moneda de curso legal pero manteniendo sus respectivas monedas nacionales en circulación. Sin embargo, estos dos países están recientemente considerando la dolarización formal o total. De igual forma, varios países de América Latina han estado debatiendo la adopción del dólar como moneda oficial; la República Dominicana no ha estado ausente de este debate y algunos opinan que la dolarización total de su economía esta cada vez más cerca.

El propósito de este trabajo es revisar las ideas concernientes al proceso de dolarización total en las economías emergentes. Específicamente, el objetivo es identificar y analizar los principales costos y beneficios teóricamente relevantes para la decisión de adoptar la dolarización oficial. En ese sentido, la sección 2 presenta las distintas modalidades de dolarización. Los principales costos y beneficios son presentados en la sección 3; y finalmente, una conclusión es provista en la sección 4.

\section{2 ¿Qué es dolarización?}

El proceso de dolarización se presenta bajo tres modalidades principales: No-oficial (de facto), semi-oficial y oficial (de jure).

Una economía está parcialmente o no oficialmente dolarizada cuando una moneda extranjera desempeña la función de la moneda nacional (reserva de valor, medio de cambio y unidad de cuenta) sin que esa moneda sea de curso legal. La literatura económica identifica tres etapas en esta modalidad de dolarización. En la primera etapa, se produce una "sustitución de activos" porque las personas mantienen bonos y depósitos en el extranjero y/o dinero en efectivo (en 
moneda extranjera) bajo el colchón como una forma de preservar su valor ${ }^{2}$.

La segunda etapa es conocida como el proceso de "sustitución monetaria". Esta etapa se caracteriza porque la personas mantienen grandes montos de monedas extranjeras depositadas en el sistema bancario doméstico (si es permitido) como reserva de valor y utilizan billetes extranjeros como medios de cambio ${ }^{3}$ aunque la moneda extranjera no sea de curso legal. En la última etapa de la dolarización parcial, las personas piensan en términos de moneda extranjera y los precios en moneda doméstica están indizados con el tipo de cambio (unidad de cuenta).

La segunda modalidad de dolarización es conocida como el sistema bimonetario o dolarización semioficial que se presenta cuando la moneda extranjera es de curso legal y puede dominar los depósitos bancarios, pero juega un papel secundario con respecto a la moneda doméstica cuando se trata de pagar salarios, impuestos, obligaciones domésticas del gobierno y transacciones del día a día (electricidad, gas, agua, etc. $)^{4}$.

Por último, la dolarización de jure (dolarización oficial o total dolarización) puede ser definida como una situación en la cual un país abandona su propia moneda y adopta la moneda de otro país. La moneda extranjera tiene el exclusivo estatus

2 La personas hacen esto porque ellos quieren protegerse de la pérdida de bienestar ocasionada por la inflación o la devaluación de la moneda doméstica. Asimismo, podrían querer evitar los actos de confiscación o congelamiento de los depósitos que algunos países han experimentado. En ese orden, el monto involucrado es difícil de calcular porque incluye los billetes en moneda extranjera que las personas guardan debajo del colchón.

3 Es importante señalar que los salarios, impuestos y las transacciones diarias como la factura eléctrica siguen siendo pagadas en moneda doméstica, pero artículos caros (automóviles y casas) algunas veces se pagan en moneda extranjera.

4 Los países con dolarización semioficial mantienen su banco central para que conduzca su propia política monetaria. 
de curso legal ${ }^{5}$, utilizándose tanto para los contratos entre partes privadas como para los pagos del gobierno. ${ }^{6}$ La dolarización de jure tiene tres formas diferente: unilateral, bilateral y la unión monetaria.

La dolarización unilateral ocurre cuando un país decide adoptar la moneda de otro país sin un acuerdo formal o informal de aprobación del país que imprime la moneda que se adoptó. La dolarización unilateral tiene la ventaja de que puede ser implementada inmediatamente, sin tener el gobierno que gastar tiempo en negociación para llegar a un acuerdo con el gobierno que imprime. En cambio, la dolarización bilateral implica adoptar la moneda extranjera pero haciendo un tratado con el país dueño de la moneda que especifíque las condiciones y alcance de la adopción. Esto último puede implicar un largo y costoso período de negociación con el gobierno del país dueño de la moneda y su banco central. Sin embargo, un beneficio importante de establecer un tratado con el gobierno del país dueño de la moneda que se adopta o con su banco central es que incrementa la credibilidad de la medida. Ello, porque la dolarización unilateral podría ser revertida cambiando la ley que se requiera y convenciendo al público para que acepte una nueva moneda nacional introducida por su propio banco central. En cambio, la dolarización bilateral además requiere cambiar el acuerdo o tratado con el país que imprime la moneda, aumentando el costo de revertir la medida, lo cual le da un carácter más permanente. Debemos señalar que los grandes beneficios de la dolarización

5 La mayoría de los países dolarizados le dan solamente a una moneda extranjera el completo estatus de curso legal, pero Bogetic (2000) señala que Andorra se los dió al franco francés y a la peseta española (actualmente ambas monedas forman parte del Euro).

6 Si la moneda doméstica existe, ésta es confinada a un papel secundario dado que solamente se acuñarán monedas metálicas con pequeño valor. 
oficial como un todo se derivan de la credibilidad, precisamente porque es casi irreversible ${ }^{7}$.

La última forma en que se presenta una dolarización de jure es la unión monetaria. Los acuerdos bilaterales de dolarización podrían ser considerados como una medida intermedia entre la dolarización unilateral y una unión monetaria. La unión monetaria implica la necesidad de desarrollar un marco institucional y la creación de un banco central o incorporar miembros del país oficialmente dolarizado en el comité o junta monetaria del banco central propietario de la moneda que se adopta. Si analizamos los procesos de dolarización unilateral y bilateral podremos ver que en estos últimos el propietario de la moneda ancla no entrega la independencia de su política monetaria al país dolarizado, además no implican la creación de una nueva autoridad monetaria (como en el caso de la Unión Monetaria Europea). En el caso de los países que han adoptado el dólar de los Estados Unidos, la FED ha continuado siendo la exclusiva autoridad encargada de diseñar la política monetaria para el dólar y hasta ahora el gobierno de los EE.UU. no ha establecido ningún acuerdo con los países dolarizados.

En la siguiente sección se analizan teóricamente los costos y beneficios que aportaría la dolarización oficial en las economías emergentes.

\section{Costos y beneficios de una dolarización oficial}

La evaluación de si la dolarización oficial es apropiada para las economías emergentes puede ser valorada a través

7 Ecuador adoptó una dolarización unilateral, sin embargo, la adopción del dólar de los Estados Unidos en ese pais es un ejemplo significativo en que la credibilidad fue un factor crucial y donde renunciar a la moneda nacional fue visto como la única vía para realizar políticas macroeconómicas más razonables. 
del análisis de costos y beneficios, pero desafortunadamente hay datos muy limitados sobre esto, dado que sólo se tiene el caso de Panamá con un largo período de dolarización. En consecuencia, el análisis se basa fundamentalmente en términos teóricos, aunque se presentan algunos resultados empíricos.

Por otro lado, en esta sección el término de dolarización total u oficial será usado para referirnos a la dolarización tanto unilateral como bilateral. En el siguiente punto se analiza el impacto sobre la inflación y la estabilización de dolarizar oficialmente la economía.

\subsection{Inflación y estabilización}

En las últimas dos décadas, varios países emergentes han experimentado altas y volátiles tasas de inflación, lo cual los llevó a asumir programas de estabilización basados en el tipo de cambio. Sin embargo, los mismos han sido interrumpidos por la pérdida de credibilidad, lo que a su vez ha desencadenado nuevos procesos inflacionarios. En este sentido, la decisión de adoptar una moneda de un país con baja tasa de inflación compra credibilidad. La credibilidad de una dolarización oficial promueve la estabilización de precios porque induce a baja variabilidad y a bajas tasas promedio de inflación doméstica. En consecuencia, la tasa de inflación del país dolarizado converge hacia la tasa de inflación del país dueño de la moneda. De esta forma, el país que adopta el dólar de los Estados Unidos está importando la credibilidad de su autoridad monetaria y, por tanto, alcanzará bajas tasas de inflación ${ }^{8}$.

En ese sentido, el trabajo de Edwards (2001) aporta una fuerte evidencia empírica. Dicho autor compara datos de paí-

8 La tasa promedio de inflación en Panamá fue 3.1\% entre 1970-2000, mientras que para el resto de América Latina fue $15.3 \%$ en el mismo período. 
ses no dolarizados y dolarizados, mostrando que los países dolarizados han experimentado una significativa baja tasa de inflación. Esto se explica por la ausencia de un exceso de oferta monetaria y por la imposibilidad de que el gobierno pueda financiar su déficit imprimiendo dinero (Moreno-Villalaz, 1999). Como consecuencia, la ausencia de dinero producido domésticamente significa que el gasto público está efectivamente restrigindo a la disponibilidad de recursos financieros (dura restricción presupuestaria).

Por otro lado, el hecho de alcanzar una baja tasa de inflación tiene un beneficio adicional porque contribuye al incremento del ahorro y los préstamos de largo plazo".

En cuanto a la estabilización económica, Mendoza (2000) muestra teóricamente que la ganancia en bienestar, de eliminar la incertidumbre sobre la estabilización se encuentra en un rango entre 6.4 y 9.7 por ciento del nivel de consumo en estado estacionario. Sin embargo, debemos destacar que la estabilidad prometida por la dolarización es en sí misma relativa, dado que el dólar de los Estados Unidos fluctúa contra otras monedas comerciables (euro, yen y libra esterlina) y si la economía oficialmente dolarizada tiene un porcentaje importante de su deuda externa en euro, una depreciación del dólar ante el euro incrementará el valor de su deuda externa. Por el contrario, si el volumen de comercio con la euro zona o Asia es importante, el país oficialmente dolarizado perderá competitividad ante una apreciación del dólar con respecto al euro o el yen. Por otro lado, la dolarización oficial elimina los shocks de oferta monetaria doméstica pero no puede eliminar los shocks monetarios que se producen en el país ancla.

9 Con bajas tasas de inflación los jubilados y las personas con ingresos fijos pueden tener más seguridad de que sus ahorros mantienen su valor a través del tiempo. 


\subsection{La devaluación y las tasas de interés}

Con la dolarización oficial de la economía se elimina el riesgo de devaluación y, por tanto, también se eliminan los costos asociados a la volatilidad del tipo de cambio nominal. En consecuencia, se producirá una importante reducción en el costo de capital (reducción en la tasa de interés doméstica) ${ }^{10}$. La lógica es la siguiente, cuando la autoridad pierde la habilidad para usar la tasa de cambio de una forma discrecional, al mismo tiempo se reduce el riesgo de devaluación lo que conlleva a la reducción de la incertidumbre en el ambiente económico. Esto último inducirá a los agentes económicos a reducir la tasa a la que ellos descuentan los retornos futuros. Adicionalmente, con la reducción del riesgo de devaluación, se reducirá el premio por riesgo país de los préstamos internacionales ${ }^{11}$.

De esta forma, Corbo (2002) destaca que los beneficios de una dolarización oficial para países de Centroamérica y el Caribe están derivados de la baja tasa de interés resultante de la eliminación de los riesgos monetarios y sus premios asociados. Asimismo, Powell y Sturzenegger (2000) afirman que la eliminación de los riesgos monetarios tendrán un impacto signiticativo sobre el riesgo país en los países emergentes latinoamericanos. Por el contrario, el trabajo de Edward (2001) destaca que los costos de capital de Panamá en el mercado internacional no han sido los más bajos en América Latina. En efecto, este autor muestra que los márgenes (spreads) de los bonos panameños han sido sistemáticamente más altos que el spread sobre los bonos soberanos de Chile con igual madurez.

10 Sin embargo, cambios en el tipo de cambio real podrían continuar, lo cual introduciria diferencia entre la tasa de interés doméstica y la externa (ver Fontaine y Vergara, 2000).

11 Ver a Berg y Borensztein (2000). 
Es interesante destacar que Chile es un país que en los últimos años ha experimentado un incremento en el grado de flexibilidad de su tipo de cambio.

En ese mismo sentido se inscribe el trabajo de Goldfajn y Olivares (2000). Dichos autores análizan tres países latinoamericanos ${ }^{12}$, encontrando que la eliminación del riesgo monetario debería implicar bajas tasas de interés pero no necesariamente bajos spreads sobre los bonos de deudas denominados en moneda extranjera y que la dolarización no garantiza el acceso automático al mercado internacional de capital. Para estos autores, aunque los spreads de Panamá son relativamente bajos comparados con el promedio de América Latina, ellos no son más bajos que los de Costa Rica (que tiene un tipo de cambio flexible). De acuerdo a su análisis, no está claro si la reducción de la tasa de interés doméstica en Panamá es consecuencia de la dolarización total de su economía o por la competitividad internacional de su sistema bancario.

\subsection{La política fiscal}

Por el lado de la política fiscal, la pérdida de señoriaje es uno de los costos más importante y controversiales de la dolarización oficial. La recaudación por señoriaje viene con el poder que tiene la autoridad para imprimir dinero y depende de la tasa de crecimiento del producto y de la tasa de inflación del país. Uno puede considerar el costo de perder el señoriaje como un costo de capital (stock) realizado por una vez (el costo de comprar la base monetaria) o, de forma equivalente, como un flujo contínuo (la pérdida flujo del señoriaje) ${ }^{13}$.

12 Argentina (convertibilidad), Costa Rica (tipo de cambio flotante) y Panamá (totalmente dolarizado).

13 Ver Fischer (1982) 
Para el caso de las economías más grandes de América Latina (Argentina, Brazil, Chile y México) Morandé y Schmidt-Hebbel (2000) estiman esas pérdidas entre $2.2 \%$ y $4.4 \%$ del PIB en 1999 para el primer componente y entre $0.12 \%$ del PIB y $0.25 \%$ del PIB para el segundo. Debemos señalar que bajas tasas de inflación conducen hacia un bajo costo flujo. Además, si el país oficialmente dolarizado ha establecido un acuerdo o una dolarización bilateral para recibir parte de las ganancias por señoriaje ${ }^{14}$, entonces el costo flujo neto disminuye. Para los países de Centroamérica y el Caribe la pérdida por el primer componente representa alrededor del $1 \%$ del PIB, una cantidad significativa si se mira como porcentaje de las recaudaciones fiscales en un año normal (Hausmann y Powell, 1999). Pero, la pérdida de señoriaje puede ser contrarrestada con la reducción del costo del servicio de la deuda pública que resulta de la reducción en la tasa de interés y este factor seguramente es más significativo que el $1 \%$ del PIB en pérdida de señoriaje (Dornbusch, 2001).

Por otro lado, Schmitt-Grohé y Uribe (1999) argumentan que cuando los países no toman en cuenta el crecimiento de la base monetaria a través del tiempo debido al crecimiento del producto y la inflación, se están subestimando los ingresos por señoriaje en un rango desde $-50 \%$ hasta $1,000 \%$ dependiendo del valor de la tasa de inflación y de la tasa real de crecimiento de la economía ${ }^{15}$. Sin embargo, si analizamos las razones por la que los países consideran la opción de una

14 Podemos señalar dos precedentes: primero, la regla para compartir señoriaje negociada por los países miembros del Euro-11 y segundo, la formula fija adoptada en el acuerdo entre las autoridades de Sudáfrica y Namibia. Hasta el momento, EE.UU. no tiene un acuerdo de compartir el señoriaje con Panamá ni con ningún otro país oficialmente dolarizado.

15 De acuerdo a estos autores, el ingreso por señoriaje solamente estará sobrestimado en el irrealístico caso en que la tasa de inflación es menor que una tasa de crecimiento real negativa. 
dolarización oficial podemos ver que el factor común es crear un ambiente económico más estable que motive la inversión y el crecimiento. Si ello se cumple, entonces el incremento en la recaudación tributaria, debido al incremento en la actividad económica, debería compensar, a lo menos parcialmente, la pérdida de ingreso por señoriaje. Además, la pérdida neta del señoriaje es mínima en algunos países dado que inician su dolarización oficial desde una alta dolarización parcial. En consecuencia, el costo de comprar la base remanente de la moneda doméstica debería ser pequeño ${ }^{16}$.

En otro orden, un factor importante que debe tomarse en cuenta es el grado de desarrollo o eficiencia del sistema tributario en las economías emergentes. Si un país adopta oficialmente la dolarización (importando bajas tasas de inflación) pero mantiene el nivel de gasto del gobierno, tendrá que incrementar los impuestos lo que producirá una pérdida de bienestar en los consumidores. Pero al mismo tiempo, si hay una alta economía subterránea o alta evasión tributaria la autoridad verá limitada las recaudaciones del sector público y precisará de recortes en el gasto o el establecimiento de otras fuentes de ingresos tributarios (potencialmente más distorsionadores). Asimismo, si dicho país históricamente ha financiado su déficit presupuestario con una combinación de deuda y emisión monetaria, entonces, si el costo marginal de incrementar las recaudaciones a través de un incremento de los impuestos excede el costo marginal de incrementar las recaudaciones a través de la inflación (señoriaje e impuesto inflación), sería óptimo reducir los impuestos e incrementar la inflación (abandonando la dolarización o no dolarizando). En consecuencia, países con un ineficiente sistema tributario y experiencia de

16 Para un pais que no tenga suficiente reservas internacionales para reemplazar la moneda doméstica en circulación, la compra del stock inicial podría añadir costos indirectos. 
altos costos de incrementar las recaudaciones a través de un incremento en la tasa de impuesto, encontrarán más ventajoso incrementar las recaudaciones a través de un incremento de la inflación. Sin embargo, el financiamiento del déficit a través de la inflación puede ser una ventaja para los gobiernos, pero no hay evidencia de que lo sea para el país en general.

\subsection{Integración Comercial}

Un beneficio potencial de la dolarización oficial puede ser el incremento en el nivel de integración comercial del país dolarizado con el país dueño de la moneda. El uso de la misma moneda facilita el comercio en bienes y servicios, dada la reducción de los costos de transacciones relacionados con los cambios de moneda. Rose y Engel (2002) señalan que los miembros de una unión monetaria tienen más comercio y menos volatilidad del tipo de cambio real que los países con sus propias monedas. Ellos añaden que los ciclos económicos están más altamente sincronizados entre países que forman parte de una unión monetaria que entre países con sus propias monedas. De acuerdo a estos autores, la magnitud del efecto del comercio bilateral en economías dolarizadas es de $240 \%$. Resultados similares son encontrados en el trabajo de Frankel y Rose (2000). Su trabajo sugiere que una unión monetaria conduce a un significativo incremento del comercio bilateral (290\%). Asimismo, Levy Yeyati (2001) muestra que el comercio bilateral tiene un impacto positivo $(65 \%)$ cuando se utiliza una moneda común.

Por el contrario, Thom y Walsh (2002) muestran que la tasa de crecimiento del comercio entre el Reino Unido y la República de Irlanda no declinó significativamente como resultado del fin del vínculo monetario entre estas dos economías en el año 1979. En ese mismo sentido, Klein (2002) presenta evidencia 
de que el efecto de una dolarización total sobre el comercio con los EE.UU. no es estadísticamente diferente del efecto del tipo de cambio fijo sobre el comercio con los EE.UU.

\subsection{Crecimiento económico e inversión}

Entre los beneficios de la dolarización oficial citados por la literatura económica se destaca que la total dolarización trae consigo grandes montos de inversión extranjera y altas tasas de crecimiento económico. Con la eliminación del comercio con dos monedas diferentes se reducen costos microeconómicos y fricciones del mercado que deberían resultar en un incremento de la inversión extranjera directa y, en el mediano y largo plazo, del crecimiento económico. Adicionalmente, no habrá abruptas salidas de capital motivadas por el miedo a una devaluación. Asimismo, Moreno-Villalaz (1999) argumenta que el acceso a los mercados internacionales de capital incrementa la disponibilidad de recursos, que permiten que el nivel de inversiones sea independiente de, y no limitado por, el ahorro doméstico.

Sin embargo, la evidencia mostrada en el trabajo de Edwards (2001), sugiere que países oficialmente dolarizados tienen tasas de crecimiento más bajas que los países no dolarizados y, por tanto, bajo crecimiento del PIB per cápita. En ese sentido y a la luz de la evidencia, este autor sugiere que los shocks externos resultan en grandes costos en términos de baja inversión y crecimiento económico en países oficialmente dolarizados que en los no dolarizados. Asimismo, Levy-Yeyati y Sturzenegger (2003a) señalan que en países en desarrollo los regímenes cambiarios menos flexibles están asociados con bajo crecimiento y con alta volatilidad del producto. No debemos olvidar que la dolarización es una medida extrema de tipo de cambio fijo. 
En consecuencia, no está claro que la dolarización oficial de la economía promueva la inversión extranjera y el crecimiento económico. Además, como se señaló en la sección 3.2 la total dolarización no garantiza el acceso a lo mercados internacionales de capital.

\subsection{El prestamista de última instancia}

En la literatura económica un argumento común en contra de la dolarización oficial es la pérdida del papel del banco central local como prestamista de última instancia. En un país oficialmente dolarizado no existe un banco central con la capacidad para imprimir dinero en caso de tener que rescatar instituciones financiera con problemas de liquidez. Sin embargo, actualmente los rescates financieros raramentes son financiados imprimiendo dinero, ellos son financiados con la emisión de deuda pública interna o externa y esto es posible de hacerse en una economía oficialmente dolarizada. Asimismo, los costos de perder el prestamista de última instancia dependen fundamentalmente del nivel de dolarización parcial que tenía la economía al iniciar el proceso de dolarización oficial. Ello, porque en una economía donde un alto porcentaje de los depósitos financieros están dolarizados, el banco central tiene significativos obstáculos para proveer seguro de liquidez al sector bancario (independientemente del régimen cambiario). Además, el papel de prestamista de última instancia que asumen los bancos centrales pueden crear altos costos en término de riesgo moral (moral hazard). En cambio, un país oficialmente dolarizado puede establecer algún mecanismo que provea liquidez sin tener que incurrir en los costos de moral harzard del prestamista de última instancia.

Con respecto a lo anterior, una solución es crear un fondo de estabilización y luego establecer un acuerdo para líneas 
de crédito con bancos extranjeros. Por otro lado, está el sistema panameño en el cual el país es parte de una gran fuente de recursos internacionales. Ello, porque dada la libertad del mercado de capitales, el ajuste de portafolio de los bancos comerciales es el principal mecanismo de prestamista de última instancia (Moreno-Villalaz, 1999). En otras palabras, el rápido acceso a fondos extranjeros ofrece a los países oficialmente dolarizados un sustituto para la función del banco central como prestamista de última instancia. Entonces, si los países oficialmente dolarizados quieren obtener mayores beneficios de la total dolarización, es necesaria la integración internacional del sistema bancario doméstico. Para lograr esto, los países emergentes que consideran la dolarización oficial como una opción real deben realizar una reforma financiera antes de adoptar el dólar como moneda de curso legal.

Por otro lado, Fischer (1999) propone crear un prestamista de última instancia internacional para mitigar los efectos del pánico financiero. De acuerdo a su análisis el Fondo Monetario Internacional podría jugar ese rol ${ }^{17}$.

\subsection{La política monetaria}

La pérdida de flexibilidad en la política monetaria implica que el gobierno pierde una importante herramienta para la estabilización. La política monetaria puede reducir los shocks en la economía cuando esos shocks son anticipados oportunamente por la autoridad. En cambio, en una economía oficialmente dolarizada el gobierno no puede devaluar la moneda o financiar el déficit presupuestario mediante la creación de inflación porque no emite dinero. Sin embargo, debemos destacar que la

17 Para una crítica a esta propuesta se recomienda ver a Schwartz (1999) 
eficiencia de la política monetaria como un instrumento anticíclico (o como una forma de influir en la demanda agregada) estará limitada si en la economía existe una alta sustitución entre la moneda local y la extranjera. Además, la capacidad para usar bien los instrumentos monetarios depende de la asimetría de información entre la autoridad y el mercado.

Por otro lado, muy al contrario de lo que sostiene la justificación teórica a favor de la banca central, las mayores flexibilidades en política monetaria ocurridas en algunos países de América Latina se han traducido en más volatilidad de las tasas de interés, en vez de menos, en respuesta a cambios en las tasas de interés en los Estados Unidos. Todo lo anterior, sugiere que no tener un banco central es en verdad un beneficio más que un costo.

Es interesante destacar, que una dolarización oficial no necesariamente implica la total desaparición de la política monetaria, pero sí que habrá una drástica disminución de la capacidad de la autoridad monetaria para ejecutar su política. En Panamá todavía se acuñan monedas fraccionarias de Balboas, siendo las monedas fraccionarias una parte de la oferta monetaria $^{18}$. La razón es simplemente técnica, acuñar monedas fraccionarias domésticamente evita los gastos en que se incurrirían si se tienen que embarcar monedas desde el país emisor.

No obstante, los costos de perder la independencia monetaria se incrementan cuando los ciclos económicos entre ambos países no están correlacionados. Es decir, el país dueño de la moneda puede restringir la política monetaria (durante un auge) pero el país oficialmente dolarizado podría estar nece-

18 El hecho de acuñar monedas fraccionarias no daña la credibilidad de la dolarización oficial. 
sitando una política monetaria expansiva (porque está en una recesión). En este sentido, Alesina and Barro (2001a) argumentan que una unión monetaria o total dolarización puede (aunque no necesariamente) incrementar la integración de los países miembros y por tanto, más movimientos sincronizados del producto.

\subsection{Otros costos y beneficios}

Algunos economistas señalan que al adoptar la dolarización oficial hay altos costos asociados con las rigideces de precios y salarios nominales, lo que se tranforma en mayores tasas de desempleo y alto grado de histéresis.

Otros costos involucrados en el proceso de dolarización total, que ocurren una sola vez, son los costos de convertir precios, programas de computación, cajas registradoras y máquinas vendedoras automáticas de moneda doméstica a moneda extranjera, los cuales varían considerablemente de un país a otro ${ }^{19}$. Asimismo, hay costos legales y financieros asociados con la revisión de contratos o refinanciamientos. Finalmente, hay un costo político cuando los habitantes desean mantener la moneda doméstica como un elemento de identidad nacional. Sin embargo, acuñar monedas fraccionarias es una solución potencial a este último problema. No obstante, existe un riesgo político de la dolarización pues no debemos olvidar la dolorosa experiencia panameña durante el período de sanciones estadounidenses (1987-89) y el conflicto armado con los Estados Unidos (1989). ${ }^{20}$

19 En economías con alta inflación esto puede ser un beneficio neto más que un costo, dado que disminuirá la frecuencia necesaria para revisar los precios.

20 Hubo una caída de $25 \%$ en la oferta monetaria y de $16 \%$ en el PIB real. Asimismo, se registró un colapso en el sector construcción y el desempleo llegó al 16\%, más del doble de su nivel de los años setenta. 
Por el lado de los beneficios, podemos destacar que bajo un sistema de total dolarización los fondos de pensiones y los ahorros privados se protegerán contra la devaluación y la inflación.

\section{Conclusión}

Desde el punto de vista de la literatura, podemos concluir que la magnitud de los costos y beneficios de una $d o-$ larización oficial no serán iguales para todos las economías emergentes. La magnitud dependerá de la característica particular de cada economía, así como también de su relación con el país propietario de la moneda y con otros países oficialmente dolarizados con la misma moneda. Es importante destacar que la dolarización de facto o dolarización parcial juega un papel clave para determinar los verdaderos costos de la dolarización oficial. La dolarización total trae consigo importantes beneficios, como son la reducción de la habilidad del gobierno para generar sorpresas inflacionarias y la reducción en las tasas de interés doméstica, pero no es claro que la dolarización oficial promueva el crecimiento económico a pesar de que se estiman efectos positivos en el comercio bilateral. Además, si la inflación es parte de un esquema óptimo de impuesto para algunas economías emergentes, la disminución de las recaudaciones con la eliminación de la emisión monetaria llevará a la creación de otros impuestos que pueden reducir el bienestar de los agentes económicos. La ausencia del prestamista de última instancia implicará establecer un mecanismo que permita rescatar instituciones financieras con problemas de liquidez, lo que conlleva a realizar reformas al sistema bancario doméstico, así como una mejor supervisión del mismo. El grado de correlación de los ciclos económicos 
entre el país oficialmente dolarizado y el país ancla juega un papel fundamental para determinar el costo de perder la política monetaria.

\section{Referencias}

Alesina, Alberto and Robert J. Barro (2001a), "Dollarization". The American Economic Review, Volume 91, No.2.

Alesina, Alberto and Robert J. Barro (2001b), "Currency Unions". Hoover Institution Press. United States.

Antinolfi, Gaetano and Todd Keister (2001), "Dollarization as a Monetary Arrangement for Emerging Market Economies". Federal Reserve Bank of St. Louis Review. Vol.8, No.6, pp29-39. November/December.

Beckerman, Paul (2001), "Dollarization and Semi-Dollarization in Ecuador". World Bank Working Paper 2643.

Berg, Andrew and Eduardo Borensztein (2000), "The Pros and Cons of Full Dollarization". IMF Working Paper WP/O0/50.

Bogetic, Zeljko (2000), "Official Dollarization: Current Experience and Issues". Cato Journal. Vol.20, No.2. Fall.

Broda, Christian and Eduardo Levy-Yeyati (2002), "Dollarization and the Lender of Last Resort". Mimeo. Universidad Torcuato Di Tella.

Calvo, Guillermo (1999), "On Dollarization". Mimeo. University of Maryland.

Calvo, Guillermo (2000), "Capital Markets and The Exchange Rate. With Special Reference to the Dollarization Debate in Latin America". Mimeo. University of Maryland.

Chang, Roberto and Andrés Velasco (2002), "Dollarization: Analytical Issues". NBER Working Paper 8838.

Cooley, Thomas F. and Vicenzo Quadrini (2001), "The Costs of Losing Monetary Independence: The Case of Mexico". Journal of Money, Credit, and Banking, Vol.33, No.2. 
Corbo, Vittorio (2002), "Exchange Rate Regimes in the Americas: Is Dollarization the Solution?" Documento de Trabajo No.229. Pontificia Universidad Católica de Chile.

De Grauwe, Paul (2000), "Economics of Monetary Union". Fourth Edition. Oxford University Press. Oxford, United Kingdom.

Dornbusch, Rudiger (1997), "Fiscal Aspects of Monetary Integration". The American Economic Review. Volume 87, Issue 2.

Dornbusch, Rudiger (2001), "Fewer monies, better monies". NBER Working Paper 8324.

Edwards, Sebastian (2001), "Dollarization Myths and Realities". Journal of Policy Modeling. Vol.23, No.2.

Eichengreen, Barry (2001), "What problems can dollarization solve?" Journal of Policy Modeling. Vol.23, No.2.

Fischer, Stanley (1982), "Seigniorage and the Case for a National Money". The Journal of Political Economy, Volume 90, Issue 2.

Fischer, Stanley (1999), "On the Need for an international Lender of Last Resort". The Journal of Economic Perspectives, Volume 13, Issue 4. pp.85-104. Autumn.

Fontaine, Juan Andrés (2000), "Official Versus Spontaneous Dollarization?". Cato Journal. Vol.20, No.1 Spring/Summer.

Fontaine, Juan Andrés and Rodrigo Vergara (2000), “¿Debe Chile Dolarizar?". Cuadernos de Economía. Año 37, No.110.

Frankel, Jeffrey A. and Andrew K. Rose, (2000), "Estimating the Effect of Currency Unions on Trade and Output". NBER Working Paper 7857.

Goldfajn, Ilan and Gino Olivares (2000), "Is adopting Full Dollarization the solution? Looking at the evidence". Texto para Discussao No.416. Pontifícia Universidade Católica do Rio de Janeiro.

Hausmann, Ricardo and Andrew Powell (1999), "Dollarization: Issues of Implementation". Mimeo. Inter-American Development Bank.

Klein, Michael W. (2002), "Dollarization and Trade". NBER Working Paper 8879.

Levy Yeyati, Eduardo (2001), "On the Impact of a Common Currency on Bilateral Trade". Mimeo, Universidad Torcuato Di Tella. 
Levy-Yeyati, Eduardo and Federico Sturzenegger (2001), "Dollarization: A Primer". Mimeo, Universidad Torcuato Di Tella.

Levy-Yeyati, Eduardo and Federico Sturzenegger (2003a), "To Float or to Fix: Evidence on the Impact of Exchange Rate Regimes on Growth". The American Economic Review. Volume 93, No.4.

Levy-Yeyati, Eduardo and Federico Sturzenegger (2003b), "Dollarization: Debates and Policy Alternatives". The MIT Press. Cambridge Massachusetts, United States.

Mendoza, Enrique G., (2000), "On Benefits of Dollarization when Stabilization Policy is not Credible and Financial Markets are Imperfect". NBER Working Paper 7824.

McKinnon, Ronald I. (2001), "The problem of dollar encroachment in emerging markets". Journal of Policy Modeling. Vol.23, No.2.

Morandé, Felipe and Klaus Schmidt-Hebbel (2000), "Chile's Peso: Better than (just) Living with the Dollar". Cuadernos de Economía. Año 37, No. 110.

Moreno-Villalaz, Juan Luis (1999), "Lessons from the Monetary Experience of Panama: A Dollar Economy With Financial Integration". Cato Journal. Vol.18, No.3, Winter.

Niskanen, William A. (2000), "Dollarization for Latin America?". Cato Journal. Vol.20, No.1 Spring/Summer

Powell, Andrew and Federico Sturzenegger (2000), "Dollarization: The Link between Devaluation and Defaut Risk". Mimeo. Universidad Torcuato Di Tella.

Rose, Andrew K. and Charles Engel (2002), "Currency Unions and International Integration". Journal of Money, Credit, and Banking, Vol.34, No.4.

Salvatore, Dominick, James W. Dean y Thomas D. Willett (2003), "The Dollarization Debate". Oxford University Press. United Kingdom.

Schmitt-Grohé, Stephanie and Martín Uribe (1999), "Dollarization and Seignorage: How Much is at Stake?" Mimeo. University of Pennsylvania.

Schmitt-Grohé, Stephanie and Martín Uribe (2001), "Stabilization Policy and the Costs of Dollarization". Journal of Money, Credit, and Banking, Vol.33, No.2. 
Schuler, Kurt (2000), "The Basics of Dollarization", Joint Economic Committee Staff Report, U.S. Congress.

Schuler, Kurt (2002), "The Future of Dollarization in Ecuador", Instituto Ecuatoriano de Economía Política. Guayaquil.

Schwartz, Anna (1999), "Is There a Need for an International Lender of Last Resort?". Cato Journal. Vol.19, No.1, Spring/Summer.

Sims, Christopher A. (2001), "Fiscal Consequence for Mexico of Adopting the Dollar". Journal of Money, Credit, and Banking, Vol.33, No.2.

Stein, Ernesto, Ernesto Talvi, Ugo Panizza and Gustavo Márquez, (1999), "Evaluando la Dolarización: Una Aplicación a Países de América Central y del Caribe". Mimeo. Inter-American Development Bank.

Thom, Rodney and Brendan Walsh (2002), "The effect of a currency union on trade: Lessons from the Irish experience". European Economic Review, Vol.46. 